\title{
IMPORTANCE D'ANNEXES LATÉRALES ARTIFICIELLES POUR LE RECRUTEMENT EN JUVÉNILES DE POISSONS DANS UN FLEUVE AMÉNAGÉ, LE BAS-RHÔNE.
}

\author{
Y. NICOLAS et D. PONT \\ URA-CNRS 1974, équipe DESMID, Laboratoire d'Ecologie des Systèmes Fluviaux, \\ 1 rue Parmentier, 13200 Arles, France.
}

\section{RÉSUMÉ}

Au siècle dernier, des digues submersibles, délimitant des "caissons", ont été érigées dans le chenal principal du Bas-Rhône. En comparant le peuplement en juvéniles de poissons du chenal, de 10 caissons et de 2 bras morts, nous avons mis en évidence une nette distinction faunistique de ces milieux, les caissons étant qualitativement et quantitativement les plus riches. II apparaît que la richesse en juvéniles de ces biotopes dépend de la diversité d'habitats et du taux de submersion par le fleuve. Les abondances des 7 espèces principales sont influencées par au moins une des 10 variables décrivant les milieux. Les caissons jouent donc un rôle essentiel comme sites de nursery, à la place de l'ancienne plaine alluviale, et contribuent au maintien de la biodiversité piscicole de ce secteur.

Mots-clés : poisson, juvénile, habitat, nursery, fleuve, plaine alluviale, aménagement.

\section{IMPORTANCE OF ARTIFICIAL BACKWATERS FOR JUVENILE FISH RECRUITMENT IN A HEAVILY REGULATED LARGE RIVER, THE LOWER-RHÔNE.}

\begin{abstract}
During the last century, submersible dikes have been built in the main channel of the Lower Rhône River. A comparison of the juvenile fish assemblages of the channel, of 10 dike fields and 2 dead arms, showed a clear faunistical distinction between these sites ; dike fields were qualitatively and quantitatively the richest. We found that juvenile richness in these biotopes depends on habitat diversity and submersion rate. The abundances of the 7 main species were also influenced by at least one of the 10 variables that described these sites. Thus, the dike fields now play a major role as nursery sites instead of the ancient floodplain, and contribute in maintaining fish biodiversity in this area.
\end{abstract}

Key-words : fish, juvenile, habitat, nursery, large river, floodplain, management.

\section{INTRODUCTION}

Il est reconnu que l'aménagement des cours d'eau, par endiguements et enrochements des rives, provoque le plus souvent une diminution de la diversité piscicole (BROOKES, 1988). Deux raisons peuvent expliquer ce phénomène : d'une part, les endiguements enlèvent toute possibilité d'exploitation des milieux latéraux de la plaine alluviale par l'ichtyofaune, utilisation profitable voire même nécessaire à l'écologie de certaines espèces (WELCOMME, 1979), et d'autre part, l'homogénéisation des faciès de rive entraîne une perte de diversité d'habitats utilisables par différentes espèces.

Le secteur arlésien du Bas-Rhône se caractérise par la présence de compartiments (ou caissons) plus ou moins connectés au chenal. Une récente étude a montré que deux 
de ces caissons jouaient un rôle de nursery pour les juvéniles de poissons (POIZAT, 1993). Nous avons décidé d'approfondir ces premiers résultats en comparant le peuplement en juvéniles du chenal principal, de dix caissons et de deux bras morts (ou lônes). Puis nous avons tenté de déterminer les propriétés de ces annexes pouvant expliquer leur meilleure production et leur recrutement optimal en juvéniles de poissons.

\section{MATÉRIEL ET MÉTHODES}

\subsection{Le site}

Ce secteur du Bas-Rhône est situé en aval du barrage de Vallabrègues, dernier ouvrage hydroélectrique établi sur le Rhône, et s'étend sur sept kilomètres juste en amont de la défluence Grand Rhône/Petit Rhône. Le débit moyen journalier y est de $1750 \mathrm{~m}^{3} . \mathrm{s}^{-1}$ (données de la Compagnie Nationale du Rhône sur 30 ans) mais le niveau d'eau connaît d'importantes fluctuations aussi bien journalières, selon le fonctionnement de la retenue de Vallabrègues, qu'annuelles ; celles-ci atteignant six mètres entre les conditions d'étiage $\left(600 \mathrm{~m}^{3} \mathrm{~s}^{-1}\right)$ et une crue de fréquence annuelle $\left(5000 \mathrm{~m}^{3} \cdot \mathrm{s}^{-1}\right)$.

Ce secteur a été aménagé au cours du siècle dernier par des enrochements des berges, principalement en rive droite, et par l'édification de digues submersibles dans le chenal principal. Ces digues délimitent des caissons qui se distinguent par des connectivités différentes avec le chenal. Le but de ces digues était d'une part de ralentir le courant dans les parties convexes afin que les sédiments s'y déposent lors des crues et, d'autre part, d'accélérer le courant afin de surcreuser le chenal et de le maintenir navigable. Sous l'influence de cette importante sédimentation (RODITIS et PONT, 1993), des faciès variés se sont développés dans les caissons créant ainsi une diversité d'habitats favorable à l'ichtyofaune.

Sur les quatorze caissons (C-) dénombrés sur le secteur, dix ont été retenus et échantillonnés. Le chenal $(\mathrm{CH})$ et deux lônes (L-), dont une est tronçonnée en trois parties par deux seuils, sont aussi englobés dans cette étude. Chaque site a été cartographié puis défini en utilisant dix variables descriptives prenant en compte leur morphologie, leur typologie et leur hydrologie:

\section{Variables morphologiques:}

- Indice de réduction de surface (Reds) qui est le rapport entre la surface maximale en période de hautes eaux et la surface à l'étiage,

- Indice de développement du rivage à l'étiage (Rétg), calculé selon la formule : $R=\frac{P}{2 \sqrt{ } \pi . S}$
où $P$ et $S$ sont respectivement le périmètre et la surface à l'étiage.

L'utilisation de tels indices nous permet de prendre en compte totalement les différences morphologiques entre stations, tout en évitant des effets "Taille» de stations dans les traitements statisques ultérieurs.

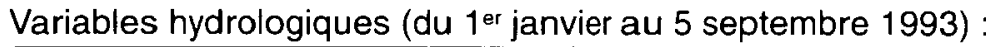

- Fréquence d'isolement (Fiso),

- Fréquence de connexion (Fcon),

- Fréquence de submersion (Fsub).

Elles caractérisent les conditions hydrauliques qui règnent dans les stations, celles-ci dépendant directement du débit du chenal. En comparant les débits moyens journaliers du Rhône à Vallabrègues avec les débits de connexion (par des passes étroites n'entraînant pas une homogénéisation des masses d'eau) et de submersion (digues noyées) déterminés sur le terrain, nous avons pu calculer pour chaque station le nombre de jours de submersion par les eaux du chenal, de connexion et d'isolement, pendant la période d'étude (248 jours). Puis, nous avons exprimé ces résultats sous forme de fréquences, la somme des trois fréquences étant égale à 1. Le chenal a été codé 1 en ce qui concerne la fréquence de submersion et 0 pour les autres. 
Variables typologiques:

- Fréquence des rives en blocs (Bloc),

- Fréquence des rives en plage de galets-graviers (Ggpl),

- Fréquence des rives de limon en pente forte (Lipf),

- Fréquence des rives en plage de limon (Lipl),

- Indice de diversité d'habitats (Dhab).

Quatre types de faciès de rives ont été définis à partir de critères physiques (pente) et granulométiques (substrat) : les rives de blocs, les plages de galets-graviers, les rives de limon en pente forte et les plages de limon. Après les avoir répertoriés sur le terrain, nous avons calculé les quatres premières variables en divisant la longueur de chacun de ces faciès de rive par le périmètre à l'étiage de chaque station. L'indice de diversité d'habitat est un indice de Shannon calculé à partir des mesures brutes de chaque faciès, il permet de prendre en compte le nombre de faciès et leur importance respective dans chaque station.

Le tableau des 15 stations (en ligne) et des 10 variables descriptives (en colonne) a été analysé par une Analyse en Composantes Principales normée (ACPn), la normalisation étant obligatoire car les variables sont de natures différentes. Puis, nous avons effectué une classification hiérarchique, en utilisant l'algorithme de moment d'ordre 2, des coordonnées factorielles des lignes de l'ACPn. Ces traitements statistiques nous ont permis de montrer que les 15 stations s'organisent selon un gradient transversal en fonction de leur typologie, de leur hydrologie et de leur morphologie, les 10 variables présentant de fortes contributions aux deux premiers axes de l'ACPn. Les stations ont alors été numérotés, de $\mathrm{C} 1$ à $\mathrm{C} 10$ pour les caissons et de L1 à L4 pour les lônes, en fonction de leur position dans ce gradient transversal (Figure 1).

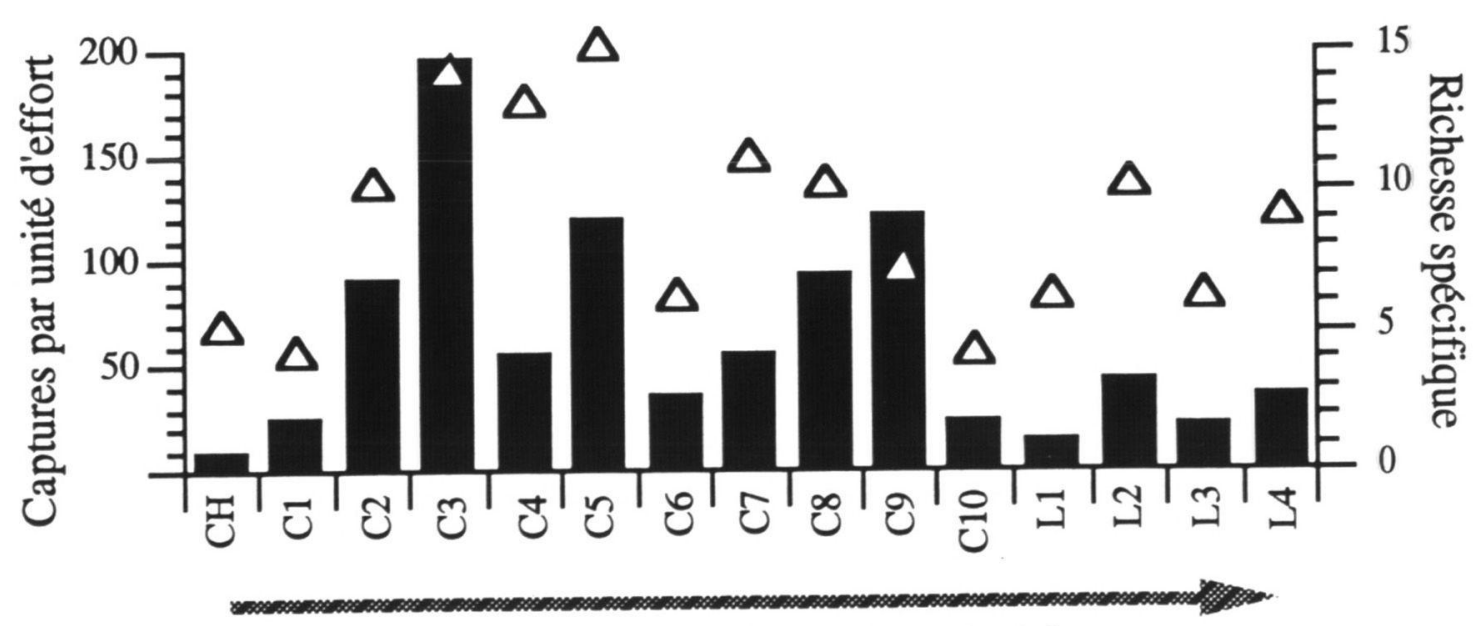

Gradient transversal de la plaine alluviale

Figure 1 : Effectifs de $0^{+}$capturés, en données corrigées ( $\left.\square\right)$, et nombre d'espèces $(\Delta)$ par station.

Figure 1 : Catch per unit effort of young fish of the year $(\square)$ and specific richness $(\Delta)$ in each station. 


\subsection{Technique et stratégie d'échantillonnage}

Nous avons adopté une méthode d'échantillonnage par pêche électrique en EPA (échantillonnage ponctuel d'abondance; NELVA et al., 1979), avec du matériel de pêche électrique portable, à partir d'une barque dans les annexes et d'un bateau dans le chenal. Nous avons utilisé une anode de dix centimètres de diamètre afin d'optimiser l'échantillonnage des juvéniles COPP (1989).

Le plan d'échantillonnage a été stratifié selon les faciès rencontrés dans chaque station. Le nombre d'EPA par station a été fixé en fonction de leur surface et seuls les faciès les plus étendus ont été échantillonnés proportionnellement à leur importance. Deux campagnes d'échantillonnage ont été effectuées pendant l'été 1993. L'espèce de chaque poisson capturé a été déterminée et tous les individus ont été mesurés au millimètre près (longueur totale) avant d'être remis à l'eau. Les effectifs en juvéniles de l'année $\left(0^{+}\right)$ont été précisés en analysant les structures en classes de taille de chaque espèce.

\subsection{Traitement des données}

Nous avons d'abord pondéré les effectifs bruts des captures par la proportion respective des faciès échantillonnés dans chaque station. Puis, afin de pouvoir comparer le peuplement en $0^{+}$de chaque station, nous avons ramené ces données à un même effort de capture, i. e. 20 EPA par station. Enfin, les effectifs ont été transformés en log $(x+1)$ pour diminuer les variances et minimiser l'effet de la non-normalité de la distribution des effectifs de poissons par station (ELLIOTT, 1977).

Afin de mettre en évidence d'éventuelles ressemblances et différences entre les abondances spécifiques de juvéniles des différentes stations, nous avons effectué une Analyse en Composantes Principales (ACP) centrée (DOLÉDEC et CHESSEL, 1992) du tableau "espèces $x$ stations». Puis, dans le but de savoir s'il existait des variables de milieu pouvant expliquer les variations faunistiques entre stations, nous avons testé par permutations (CROWLEY, 1992) l'effet de chacune des variables descriptives des stations, ces variables ayant été préalablement codées en classes afin de détecter des réponses non linéaires.

\section{RÉSULTATS}

Nous avons capturé 3004 poissons, appartenant à 22 espèces et 7 familles (Figure 2), dont $79 \%$ de juvéniles de l'année, ce qui démontre l'efficacité de notre technique d'échantillonnage des $0^{+}$. Les juvéniles de gardon, chevesne et hotu dominent largement, cumulés, ils représentent plus de $75 \%$ des effectifs bruts de $0^{+}$. Pour la première fois des spécimens de Pseudorasbora parva, Cyprinidé originaire du sud-est asiatique (ROSECCHI et al., 1993), sont recensés dans le secteur.

Les peuplements en juvéniles des différentes stations se différencient aussi bien qualitativement que quantitativement (Figure 1). Le chenal apparaît comme la station la plus pauvre en juvéniles, avec seulement cinq espèces recensées et à peine dix poissons pour 20 EPA, alors que la majorité des caissons présentent plus de dix espèces et plus de cinquante poissons pour 20 EPA ; le maximum étant atteint par le caisson C3 avec 196 poissons capturés pour 20 EPA, appartenant à 14 espèces. Quant aux stations situées dans les lônes, elles semblent assez pauvres car moins de 50 poissons pour 20 EPA y sont capturés et elles ne contiennent que de six à dix espèces.

Seuls les deux premiers axes de l'ACP du tableau de contingence ont été retenus car ils expliquent à eux seuls plus de $56 \%$ de l'inertie totale (Figure 2). Le premier axe distingue les stations où les juvéniles d'espèces rhéophiles (CHE, HOT, GOU) et de gardon sont abondants ( $\mathrm{C} 3$ et $\mathrm{C} 5$ ), des stations où ces espèces sont rares ou absentes $(\mathrm{C} 10, \mathrm{~L} 2$ et L3). Le second axe sépare le caisson $\mathrm{C} 9$ du Chenal $(\mathrm{CH})$. Le premier est riche en $\mathrm{O}^{+}$ d'espèces limnophiles (GAM et PES), de gardon et d'ablette alors que seul le gardon est présent, en faible quantité, dans le second. 


\begin{tabular}{|c|c|c|}
\hline Code & Eqpices & Fandlles \\
\hline ANG & Anguilla anguilla & Anguillidae \\
\hline BRE & Abramis brama & Cyprinidae \\
\hline $\mathbf{A B L}$ & Alburnus alburneus & $\cdot$ \\
\hline BAF & Barbus bartus & $\cdot$ \\
\hline BRB & Blicca bjoerkna & $\cdot$ \\
\hline CAS & Carassies carassius \& aurams & . \\
\hline нот & Chondrostoma nasus & - \\
\hline $\mathrm{cco}$ & Cyprinus cappio & $\cdot$ \\
\hline GOU & Gobio gobio & - \\
\hline PSR & Pseudorasbora parva & - \\
\hline $\mathrm{CHE}$ & Leuciscus cephalus & $\cdot$ \\
\hline BOU & Rhodeus sericeus amarus & $\cdot$ \\
\hline GAR & Rutilus nutilus & $\cdot$ \\
\hline ROT & Scardinius erythrophehaimus & $\cdot$ \\
\hline TAN & Tinca tinca & - \\
\hline PES & Lepomis gibbosus & Centrarchidae \\
\hline BBG & Micropterus salmoides & - \\
\hline PER & Perca fuviarilis & Percidae \\
\hline SAN & Stizostedion lucioperca & - \\
\hline $\mathrm{PCH}$ & Ictalurus melas & Ictaluridoe \\
\hline GAM & Gambusia affinis & Poecillidae \\
\hline MUP & Lisa ramada & Mugilidae \\
\hline
\end{tabular}
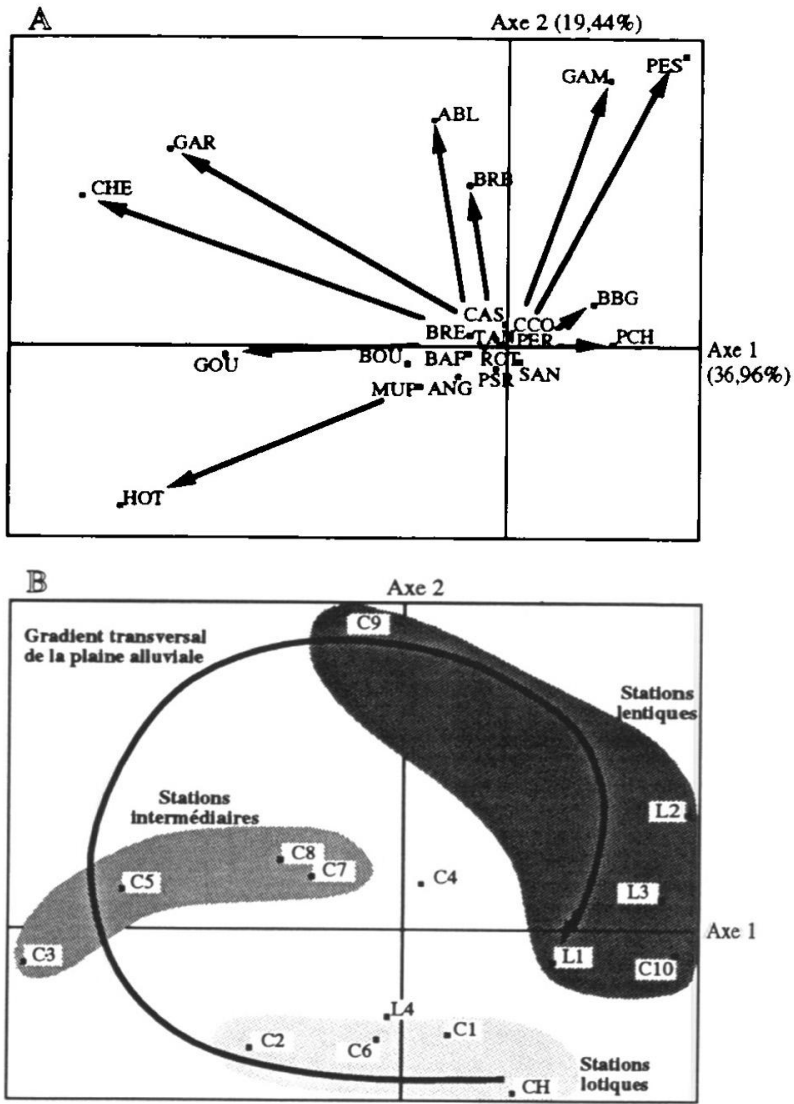

Figure 2 : ACP centrée du tableau des effectifs de $0^{+}$par station, transformé en $\log (x+1),(A)$ carte factorielle des espèces (colonnes) et (B) carte factorielle des stations (lignes) sur le plan déterminé par les axes 1 et 2.

Figure 2 : Centred PCA of the table of young fish of the year in each station after $\log (x+1)$ transformation, (A) factorial map of the species (columns) and (B) factorial map of the stations (rows) on the plan determined by axes 1 and 2 .

En résumé, si on examine le plan factoriel des lignes (stations), il semble que, plus une station est située sur la gauche, plus elle est riche en juvéniles d'espèces principalement rhéophiles. A l'inverse, les stations situées à droite semblent caractérisées par des densités moins importantes de juvéniles d'espèces limnophiles résistantes à des conditions de milieu difficiles, comme le prouve le positionnement des quatre espèces introduites d'origine américaine (PES, GAM, PCH, et BBG).

Deux des dix variables descriptives des stations ont un effet significatif sur les variations interstationnelles du peuplement global en $0_{+}$, il s'agit de la fréquence de submersion (Fsub) et surtout de la diversité d'habitat (Dhab) de chaque station. Ces deux variables expliquent respectivement $27,8 \%(p=0,0125)$ et $30,9 \%(p=0,004)$ des variations faunistiques entre stations. En ce qui concerne les variations interstationnelles d'abondance des sept espèces dominantes (Figure 3), elles trouvent toutes au moins une explication statistiquement significative parmi les variables descriptives; dans le cas du gardon, trois variables descriptives, la fréquence de submersion, la diversité d'habitats et la fréquence des rives en plage de limon (Fsub, Dhab et Lipl) ont même un effet significatif sur ses différences d'effectif entre stations. Les indices de réduction de surface et de dévelopement du rivage à l'étiage (Reds, Rétg), ainsi que les fréquences d'isolement et de connection (Fiso et Fcon) n'ont aucun effet significatif sur les variations d'abondances interstationnelles spécifiques. 

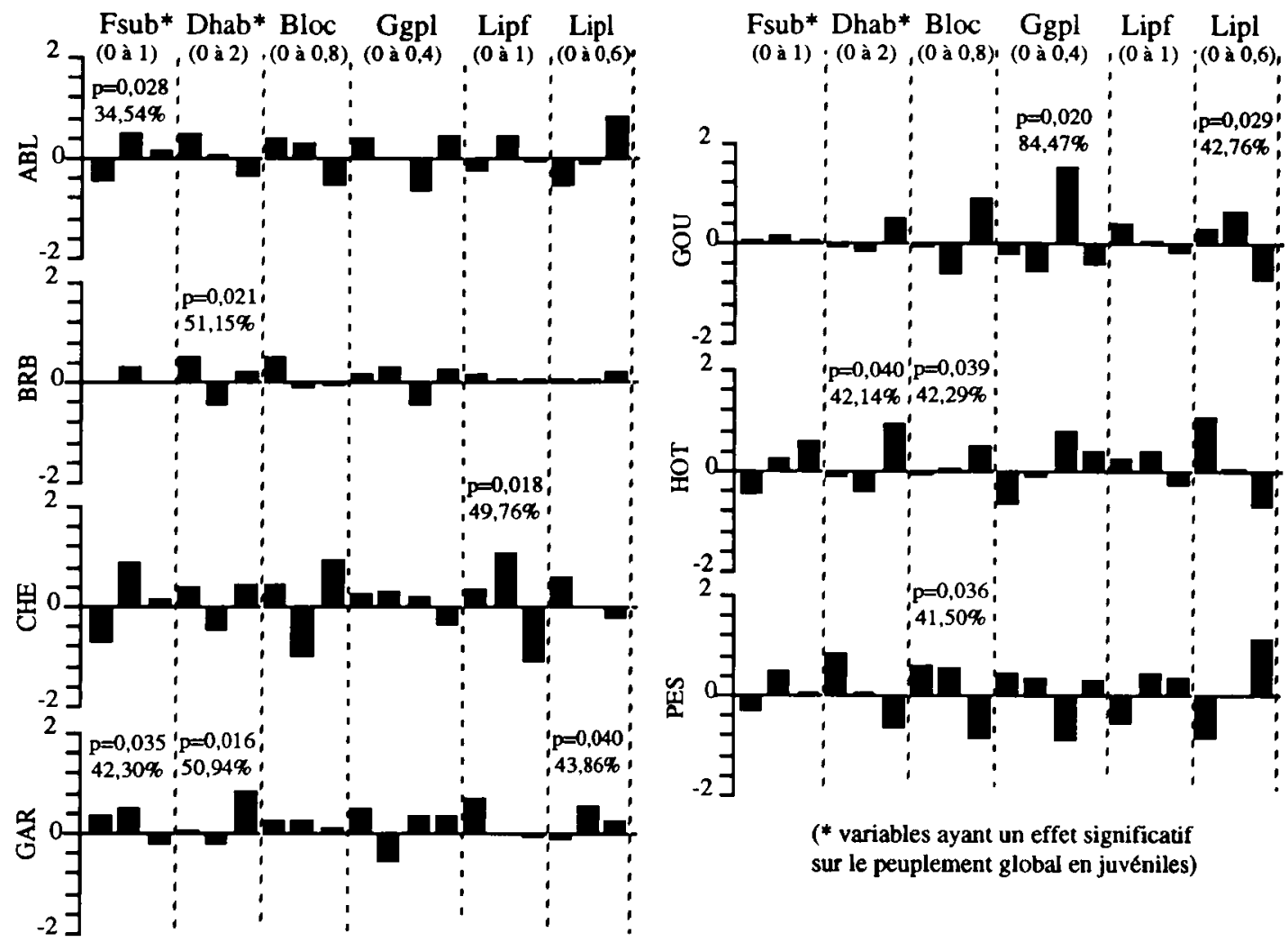

Figure 3 : Effet des variables descriptives des stations sur les 7 espèces dominant le peuplement en juvéniles. L'amplitude des valeurs de chaque variable est inscrite entre parenthèses. Les histogrammes représentent la moyenne des écarts d'abondance pour chaque modalité des variables par rapport à la moyenne générale de l'abondance en $\log (x+1)$. Le seuil de signification et le pourcentage de variance expliquée par la variable sont indiqués dans les cas où l'effet est significatif. Pour les codes des espèces, se reporter à la figure 2.

Figure 3 : Effect of each variable describing the stations on the young of the year of dominant species. The range of values for each variable is between brackets. Histograms represent the difference between the abundance of species for each category of the variables and average abundance in $\log (x+1)$. Probability and percentage of explained variation are given when the effect is significant. For species codes, see figure 2.

\section{DISCUSSION}

Cette étude corrobore les premiers résultats de POIZAT (1993) et met clairement en évidence le rôle d'une majorité des caissons comme sites de nursery pour les juvéniles de poissons. Les stations les plus riches d'un point de vue aussi bien qualitatif que quantitatif sont les caissons et principalement les caissons $\mathrm{C} 3$ et $\mathrm{C} 5$ alors que le chenal est la station la plus pauvre. Les lônes sont aussi assez pauvres tant en nombres d'espèces qu'en effectifs de juvéniles. Ces différences peuvent s'expliquer au travers du gradient transversal mis en évidence au niveau de cette plaine alluviale artificielle :

- Dans les milieux lotiques (chenal et stations typologiquement et hydrologiquement voisines), on trouve une faible quantité de juvéniles d'espèces essentiellement rhéophiles et lithophiles. 
- Les milieux lentiques, plus isolés et parfois temporaires (lônes et caissons C9 et C10), sont caractérisés par des espèces limnophiles autochtones ou par des espèces introduites résistantes aux conditions de milieu extrêmes et à stratégie de reproduction élaborée, selon BALON (1975).

- Au centre de ce gradient, on trouve la majorité des caissons qui présentent un peuplement de juvéniles diversifié à des densités importantes, constitué d'espèces rhéophiles, limnophiles et surtout ubiquistes. Toutes les stratégies de reproduction y sont représentées.

Une telle zonation des différents milieux d'une plaine alluviale selon leur peuplement en juvéniles de poissons a déjà été observée par COPP (1989) sur le HautRhône. La comparaison de nos résultats avec ceux de cet auteur tend à rapprocher une majorité des caissons des milieux parapotamiques, encore ouverts à l'aval sur le fleuve mais fermés à l'amont.

Le peuplement en juvéniles des différents milieux de cette plaine alluviale artificielle dépend de deux facteurs:

- la diversité d'habitats. Ce résultat conforte la validité de notre étude car il confirme que, plus un milieu possède une diversité d'habitats importante, plus il est exploité par un grand nombre d'espèces à preferendum d'habitats différents (GORMAN et KARR, 1978), à des fins de reproduction et/ou de nursery,

- la fréquence de submersion qui schématise du point de vue hydrologique le gradient de cette plaine alluviale (du chenal jusqu'aux lônes le plus souvent complètement isolées) et sur laquelle se juxtaposent les preferendums courantologiques des espèces.

Ainsi, la richesse qualitative et quantitative du peuplement des caissons est la résultante combinée de la diversité d'habitats et d'un taux modéré de connectivité hydrologique avec le chenal.

Les variables descriptives qui influent sur les différences de distribution spécifique entre stations, sont caractéristiques des milieux préférentiellement occupés par les juvéniles des espèces principales. La fréquence de submersion influence la distribution des juvéniles d'ablettes car ce poisson pélagique est absent des milieux les plus isolés. La distribution de la brème bordelière dépend fortement de la diversité d'habitats car cette espèce est concentrée dans les milieux à diversité d'habitats très faible (lônes) ou très forte (caissons), mais elle est absente des milieux lotiques à diversité d'habitats moyenne. Cette réponse positive aux situations extrêmes de diversité d'habitats ne semble basée sur aucune réalité biologique ; cependant, elle exprime peut-être le fait que la distribution de la brème bordelière est soumise à l'interaction de plusieurs facteurs, non significatifs pris un par un, synthétisée par la diversité d'habitats. Le chevesne est rare, voire même inexistant, dans les lônes et les caissons les plus isolés, milieux caractérisés par des rives de limon en pente forte ; l'abondance du chevesne est donc inversement corrélée à l'importance des rives de limon en pente forte d'une station. Le gardon est la seule espèce capturée dans toutes les stations. Cependant, trois variables descriptives affectent fortement son abondance. Les juvéniles de gardon se développent le mieux dans les caissons où la diversité d'habitats est maximale, où la fréquence de submersion est moyenne et où il y a suffisamment de plages de limon. Les jeunes poissons trouvent au niveau de ce faciès une nourriture abondante et de nombreux refuges car la productivité biologique et les herbiers y sont importants. De même les stations, où les plages de galets-graviers et de limon sont en quantité assez importante (caissons), sont riches en goujon, espèce benthique qui exploite préférentiellement ce type d'habitat. On trouve les juvéniles de hotu dans les caissons à tendance lotique caractérisés par une importante diversité d'habitats mais avec une forte proportion de rives de bloc car les jeunes hotus peuvent non seulement y trouver refuge, mais aussi y brouter le périphyton. A l'inverse, l'abondance des juvéniles de perches soleil est inversement corrélée à l'importance des rives de bloc. Ce faciès est caractéristique des milieux aménagés soumis à des courants importants (chenal et caissons à caractère lotique) alors que la perche soleil, espèce limnophile, colonise les milieux plus lentiques où les enrochements ont été faibles ou recouverts par du limon lors des crues. 


\section{CONCLUSION}

En définitive, les caissons par leur richesse en juvéniles jouent un rôle essentiel dans le recrutement et le maintien d'une diversité pisciaire au niveau de ce secteur du BasRhône. II est ainsi très intéressant de remarquer que des milieux d'origine artificielle sont devenus des sites d'intérêt écologique à la place de la plaine alluviale aujourd'hui disparue. De telles observations, concernant l'utilisation de gravières ou de caissons comme nursery ont déjà été faites par quelques auteurs (PENNINGTON et al., 1983 ; VALDEZ et WICK, 1983 ; SABO et KELSO, 1991). Du fait de son édification dans le chenal même, un caisson apparaît donc idoine à rétablir des zones de reproduction/nursery dans une grande rivière très aménagée, sans entraîner de baisse du niveau d'eau de la rivière comme c'est le cas des gravières. De même, de part leur position dans le lit mineur, les caissons vont évoluer de façon "naturelle" au gré des phénomènes d'érosion/sédimentation et s'intégreront alors correctement au paysage en y maintenant une forte diversité.

\section{REMERCIEMENTS}

Les auteurs tiennent à remercier la Compagnie Nationale du Rhône et l'Agence de l'Eau Rhône-Méditerranée-Corse pour le financement de cette étude, et toutes les personnes qui nous ont assistés tant sur le terrain qu'au laboratoire, particulièrement Evelyne FRANQUET. Cette étude s'inscrit aussi dans le cadre du programme du CNRS sur l'environnement (PIR-Environnement).

\section{BIBLIOGRAPHIE}

BALON E.K., 1975. Reproductive guilds of fishes and definition. J. Fish. Res. Board Can., $32(6), 821-864$.

BROOKES A., 1988. Channelized rivers, perspectives for environmental management. Wiley-Interscience, $326 \mathrm{p}$.

COPP G.H., 1989. The habitat diversity and fish reproductive function of floodplain ecosystems. Environ. Biol. Fish., 26, 1-27.

CROWLEY P.H., 1992. Resampling methods for computation-intensive data analysis in ecology and evolution. Annu. Rev. Ecol. Syst., 23, 405-447.

DOLÉDEC S., CHESSEL D., 1992. Recent developments in linear ordination methods for environmental sciences. Trends in Ecology, Research Trends publishers, India.

ELLIOTT J.M., 1977. Some methods for statistical analysis of samples of benthic invertebrates. Freshwater Biological Association Scientific Publication, $n^{\circ} 26$.

GORMAN O.T., KARR J.R., 1978. Habitat structure and stream fish communities. Ecology, 59 (3), 507-515.

NELVA A., PERSAT H., CHESSEL D., 1979. Une nouvelle méthode d'étude des peuplements ichtyologiques dans les grands cours d'eau par échantillonnage ponctuel d'abondance. C. R. Acad. Sci. Paris, Série D., 289, 679-791.

PENNINGTON C.S., BAKER J.A., BOND C.L., 1983. Fishes of selected aquatic habitats on the Lower Mississippi River. U.S. Army Engineer Waterways Experiment Station, $70 \mathrm{p}$.

POIZAT G., 1993. Echelle d'observation et variabilité des abondances de juvéniles de poissons dans un secteur aval du Rhône. Thèse de doctorat, Université Lyon I, 224 p.

RODITIS J.C., PONT D., 1993. Dynamiques fluviales et milieux de sédimentation du Rhône à l'amont immédiat de son delta. Méditerranée, 3, 4, 5-18.

ROSECCHI E., CRIVELLI A.J., CATSADORAKIS G., 1993. The establishment and impact of Pseudorasbora parva, an exotic fish species introduced into Lake Mikri Prespa (north-western Greece). Aquatic conservation : Marine and Freshwater Ecosystems, 3, 223-231. 
SABO M.J., KELSO W.E., 1991. Relationship between morphometry of excavated floodplain ponds along the Mississippi River and their use as fish nursery. Transactions of American Fisheries Society, 120, 5, 552-561.

VALDEZ R.A., WICK E.J., 1983. Natural versus manmade backwaters as native fish habitat. In V. A. L. V.D. ADAMS (Eds.), Aquatic resources management of the Colorado river ecosystem, 519-536, Annual Arbor. Science Publ.

WELCOMME R., 1979. Fisheries ecology of floodplain rivers. Longmans, London, 317 p. 